\title{
LncRNA FAM83A-AS1 facilitates tumor proliferation and the migration via the HIF-l $\alpha /$ glycolysis axis in lung adenocarcinoma
}

\author{
Zhencong Chen\#, Zhengyang Hu\#, Qihai Sui\#, Yiwei Huang\#, Mengnan Zhao, Ming Li, Jiaqi Liang, Tao Lu, \\ Cheng Zhan ${ }^{\bowtie}$, Zongwu Lin ${ }^{凶}$, Fenghao Sun ${ }^{凶}$, Qun Wang, Lijie Tan \\ Department of Thoracic Surgery, Zhongshan Hospital, Fudan University, No. 180, Fenglin Road, Shanghai, 200032, China \\ \# These authors contributed equally: Zhencong Chen, Zhengyang Hu, Yiwei Huang, and Qihai Sui. \\ $\square$ Corresponding authors: Cheng Zhan, Zongwu Lin, and Fenghao Sun; Department of Thoracic Surgery, Zhongshan Hospital, Fudan University, No. 180, \\ Fenglin Road, Shanghai, 200032, China; Phone: 86-21-64041990; Fax: 86-21-64041990; Email: czhan10@fudan.edu.cn; lin.zongwu@zs-hospital.sh.cn; \\ sun.fenghao@zs-hospital.sh.cn
}

(c) The author(s). This is an open access article distributed under the terms of the Creative Commons Attribution License (https://creativecommons.org/licenses/by/4.0/). See http://ivyspring.com/terms for full terms and conditions.

Received: 2021.09.28; Accepted: 2021.11.14; Published: 2022.01.01

\begin{abstract}
Background: Lung adenocarcinoma (LUAD), the major subtype of lung cancer, is among the leading cause of cancer-related death worldwide. Energy-related metabolic reprogramming metabolism is a hallmark of cancer shared by numerous cancer types, including LUAD. Nevertheless, the functional pathways and molecular mechanism by which FAM83A-ASI acts in metabolic reprogramming in lung adenocarcinoma have not been fully elucidated.

Methods: We used transwell, wound-healing scratch assay, and metabolic assays to explore the effect of FAM83A-AS1 in LUAD cell lines. Western blotting, Co-IP assays, and ubiquitination assays were used to detect the effects of FAM83A-ASI on HIF-1 $\alpha$ expression, degradation, and its binding to VHL. Moreover, an in vivo subcutaneous tumor formation assay was used to detect the effect of FAM83A-AS1 on LUAD.

Results: Herein, we identified FAM83A-ASI as a metabolism-related IncRNA, which was highly correlated with glycolysis, hypoxia, and OXPHOS pathways in LUAD patients using bioinformatics analysis. In addition, we uncovered that FAM83A-AS1 could promote the migration and invasion of LUAD cells, as well as influence the stemness of LUAD cells in vivo and vitro. Moreover, FAM83A-AS1 was shown to promote glycolysis in LUAD cell lines in vitro and in vivo, and was found to influence the expression of genes related to glucose metabolism. Besides, we revealed that FAM83A-ASI could affect glycolysis by regulating HIF-la degradation. Finally, we found that FAM83A-AS1 knockdown could inhibit tumor growth and suppress the expression of HIF-1 $\alpha$ and glycolysis-related genes in vivo.

Conclusion: Our study demonstrates that FAM83A-ASI contributes to LUAD proliferation and stemness via the HIF-1 $\alpha / g$ lycolysis axis, making it a potential biomarker and therapeutic target in LUAD patients.
\end{abstract}

Key words: Lung adenocarcinoma (LUAD), Metabolic reprogramming, HIF-1a/glycolysis axis, FAM83A-AS1

\section{Introduction}

Lung cancer has the second-highest morbidity and the highest mortality of all malignant tumors, with a 5 -year overall survival (OS) of only $18 \%[1,2]$. Lung adenocarcinoma accounts for nearly $40 \%$ of lung cancer cases, contributing to the majority of cases in terms of both incidence and mortality[3].

Metabolic reprogramming, a hallmark of tumor cells, is an essential characteristic as it provides the energy required for survival and maintenance of cellular function[4, 5]. Recently, several studies found that IncRNAs, a class of non-coding transcripts that are $>200$ nucleotides in length, were involved in the tumor metabolic regulation process at a transcriptional level, affecting several metabolic pathways [6, 7]. For example, Rupaimoole $R$ et al.[8] identified lncRNA NRCP as an intermediate binding 
partner between STAT1 and RNA polymerase II, which could affect metabolism in ovarian cancer. Additionally, Huang $X$ et al.[9] revealed that lncRNA LINC00842 promotes PDAC malignancy through metabolic remodeling. These findings suggest that the interaction between lncRNAs and metabolic reprogramming is valuable for exploring new potential targets in cancer therapy.

LncRNA FAM83A-AS1, which has been suggested to cause and accelerate the occurrence and development of tumors[10, 11], is transcribed from the antisense strand of the FAM83A gene located at 8q24.13. However, FAM83A-AS1's role in tumor development is not yet clearly defined, especially in metabolic reprogramming. This study is the first to identify and validate FAM83A-AS1 as a metabolism-related lncRNA that facilitates tumor proliferation and stemness via the HIF-1a/ glycolysis axis. Moreover, by performing Co-IP, ubiquitination, and metabolic assays we provided mechanistical insight into the role of FAM83A-AS1 in the pathogenesis of LUAD.

\section{Results}

\section{Identification of FAM83A-AS1 as a metabolism-related IncRNA in LUAD patients}

Previous studies have reported that the rapid growth of malignant cells demands large amounts of energy, which indicates that energy-related metabolic pathways play an important role in tumorigenesis[12-14]. Our previous work had comprehensively analyzed the metabolic heterogeneity on a pan-cancer scale and revealed that LUAD patients could be assigned to four metabolic subgroups according to the glycolysis and oxidative phosphorylation (OXPHOS) pathways[15] (Figure 1B). Additionally, differential expression analysis in LUAD patients between the high-glycolysis \& low-OXPHOS and low-glycolysis \& high-OXPHOS groups revealed that FAM83A-AS1 was significantly elevated in the high-glycolysis \& low-OXPHOS groups (Figure 1C). We next sought to investigate the association between the expression of FAM83A-AS1 and energy-related metabolic pathways (glycolysis and OXPHOS pathways). As shown in Figure 1D, we found that the glycolysis activity and OXPHOS pathways were all highly correlated with the expression of FAM83A-AS1 (Pearson's $\mathrm{R}=0.61$ for glycolysis and -0.63 for OXPHOS, respectively). Moreover, in order to explore the differential activities of pathways between low FAM83A-AS1 expression group ( $\leq$ median expression level across all samples) and high FAM83A-AS1 expression group (>median expression level across all samples) in LUAD patients, we performed GSVA. As shown in Figure 1E, energy-related metabolic pathways (including glycolysis, bile acid metabolism, and hypoxia) were the top 3 enriched pathways in high FAM83A-AS1 expression group, suggesting FAM83A-AS1 could serve as a metabolism-related lncRNA in LUAD patients.

Next, to explore the oncogenic role of FAM83A-AS1 in LUAD patients, we performed differential expression analysis in LUAD tissues and normal lung tissues of TCGA data using the edgeR package. As shown in Figure $1 \mathrm{~F}$, we observed that FAM83A-AS1 was significantly elevated in LUAD tissues. Moreover, our previous study[16] revealed that the stemness index could serve as a biomarker in LUAD. Therefore, the relationship between the expression of FAM83A-AS1 and stemness scores was also investigated in our study. Intriguingly, as shown in Figure 1G, we found that stemness scores were positively correlated with FAM83A-AS1 expression in TCGA-LUAD patients. Additionally, we also evaluated the association between the FAM83A-AS1 expression and the clinicopathological features in LUAD patients and our results revealed that the higher expression level of FAM83A-AS1 was also linked with a worse prognosis (Figure 1H). Additionally, our results also suggested that FAM83A-AS1 expression was strongly associated with TNM staging, tumor graded, tumor size, and distant metastasis (Supplementary Figure 1A and Supplementary Figure 1B). On the other hand, differential analysis in the somatic mutation distribution was also performed in our study. As shown in Supplementary Figure 1C, our study revealed distinct patterns of the mutation between the high FAM83A-AS1 expression group and low FAM83A-AS1 expression group. Overall, our results reveal that FAM83A-AS1 is a metabolism-related lncRNA in LUAD patients and is vital for the LUAD.

\section{LncRNA FAM83A-AS1 promotes the migration, invasion and stemness of LUAD cell lines under hypoxic conditions in vitro}

To investigate the possible role of lncRNA FAM83A-AS1 in lung adenocarcinoma, we used wound-healing scratch assays to determine its effect on the invasion and migration ability of A549 and H358 cell lines. We found that FAM83A-AS1 knockdown could barely reduce the migration ability of LUAD cells under a normoxia environment (Supplementary Figure 2A). However, considering the close relationship between FAM83A-AS1 and hypoxia, we repeated the above experiments under hypoxic conditions. Interestingly, results showed that knockdown of FAM83A-AS1 in both A549 and H358 cell lines significantly inhibited the invasion and 
migration abilities of LUAD treated with $1 \% \mathrm{O}_{2}$ (Figure 2A). In addition, we also performed transwell assays in normal and FAM83A-AS1 knockdown cell lines under hypoxic conditions. As expected, the results were in line with our above outcomes (Figure
2B). On the other hand, we also found that compared with lung adenocarcinoma cells that did not knock out FAM83A-AS1, the proliferation of knocked out cells was significantly inhibited (Supplementary Figure 2B).
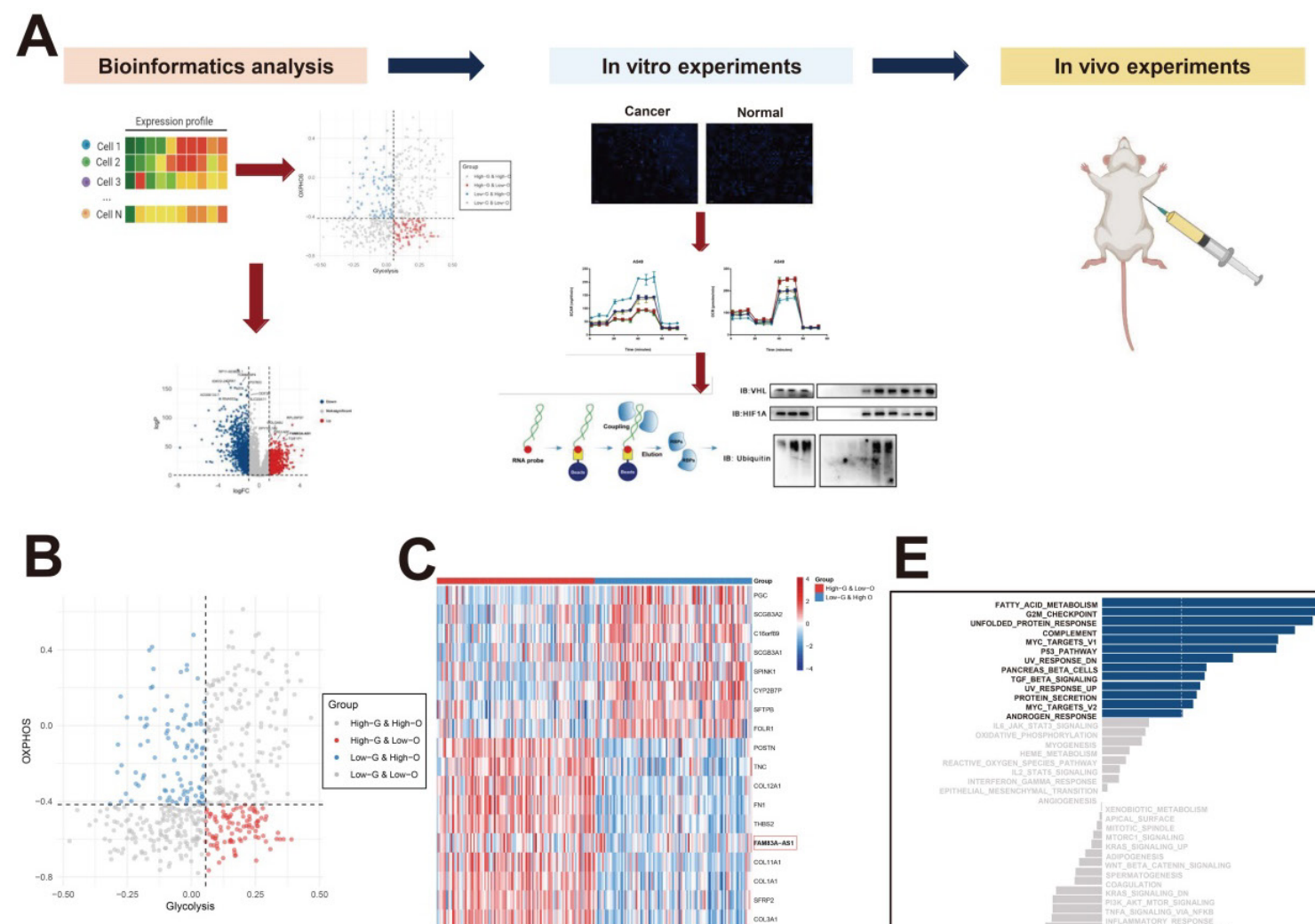

D
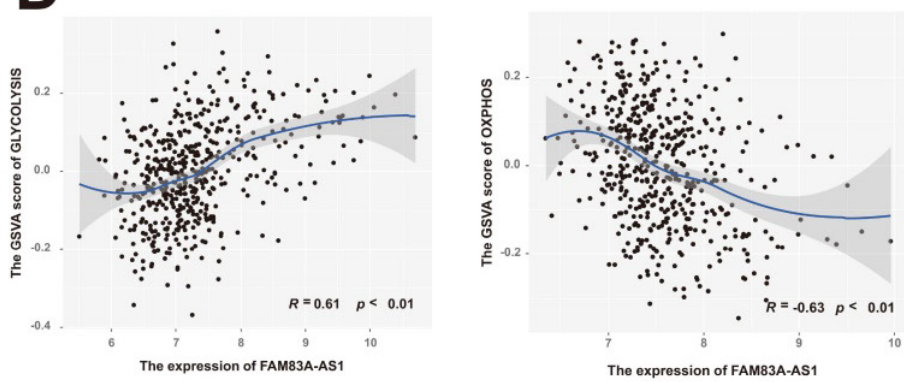

$\mathbf{F}$

G
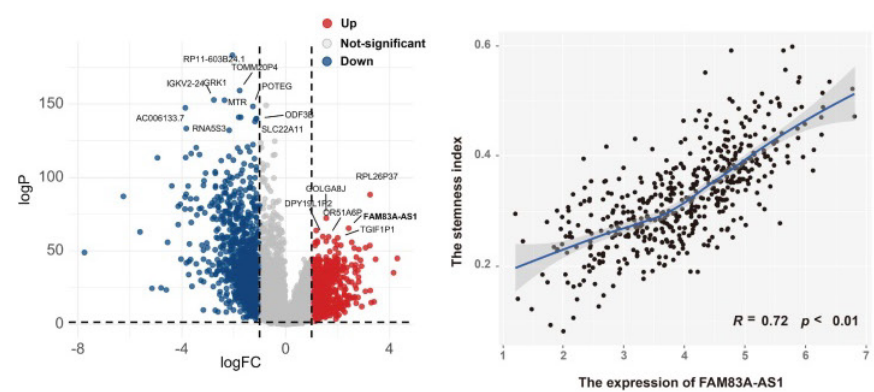

E

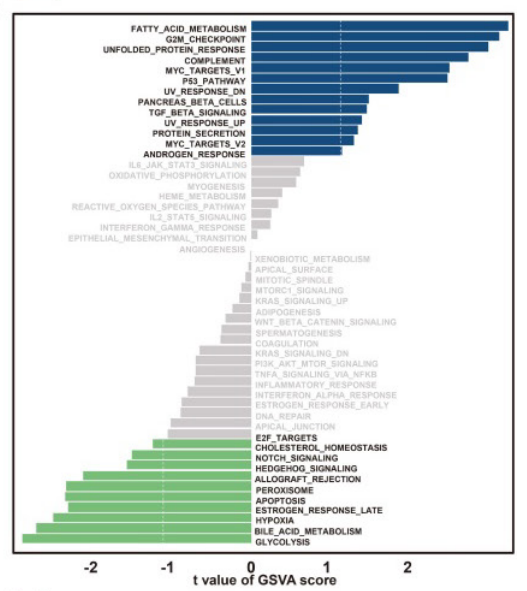

H
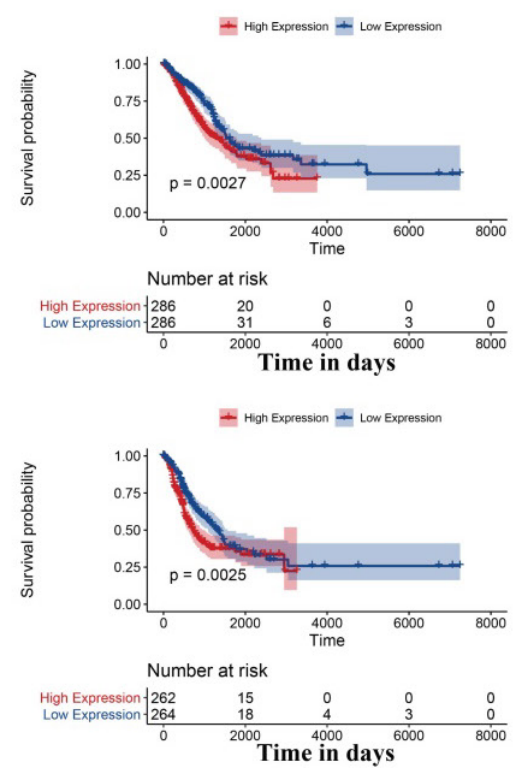

Figure 1. Identification of FAM83A-AS1 as a metabolism-related IncRNA in LUAD patients. (a) Experimental scheme for identifying FAM83A-ASI facilitated tumor proliferation and the migration via the HIF-1a/ glycolysis axis in lung adenocarcinoma. (b) The scatter plot showing the distribution of Glycolysis score (x-axis) and OXPHOS 
score (y-axis) in LUAD patients. Patients were assigned to four metabolic subgroups according to the median value of the two scores. (c) Heatmap of differentially expressed genes between high-glycolysis \& low-OXPHOS and low-glycolysis \& high-OXPHOS groups. (d) The association between the expression of FAM83A-ASI and energy-related metabolic pathways (glycolysis and OXPHOS pathways). (e) GSVA analysis of the hallmark pathways between low FAM83A-AS1 expression group ( $\leq$ median expression level across all samples) and high FAM83A-AS1 expression group (>median expression level across all samples) in LUAD patients. (f) Differential expressed genes between the LUAD and the normal lung group were shown in a volcano plot. (g) The association between the expression of FAM83A-AS1 and stemness scores. (h) Kaplan-Meyer plot of OS (up) and DFS (down) in TCGA with high or low expression group of FAM83A-AS1.

A

Oh

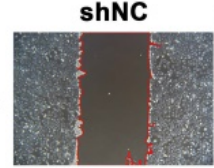

$\mathbf{2 4 h}$

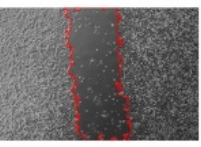

$48 \mathrm{~h}$

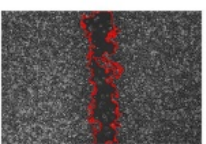

A549

ShFAM83A-AS1-1 shFAM83A-AS1-2
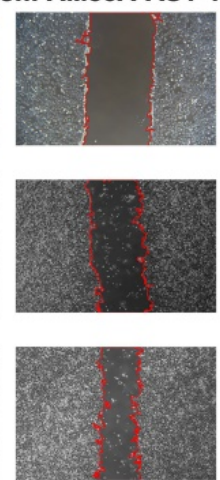

B

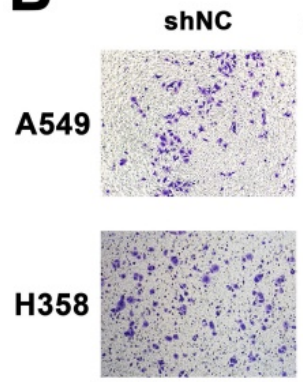

ShFAM83A-AS1-1 ShFAM83A-AS1-2

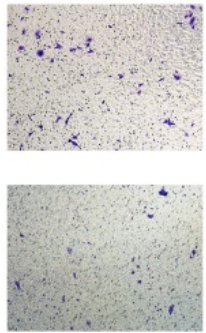

Invasion

C

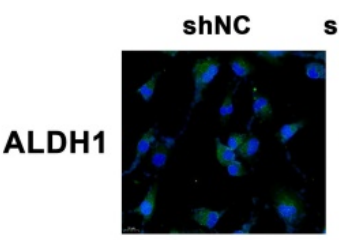

A549

ShFAM83A-AS1-1 shFAM83A-AS1-2
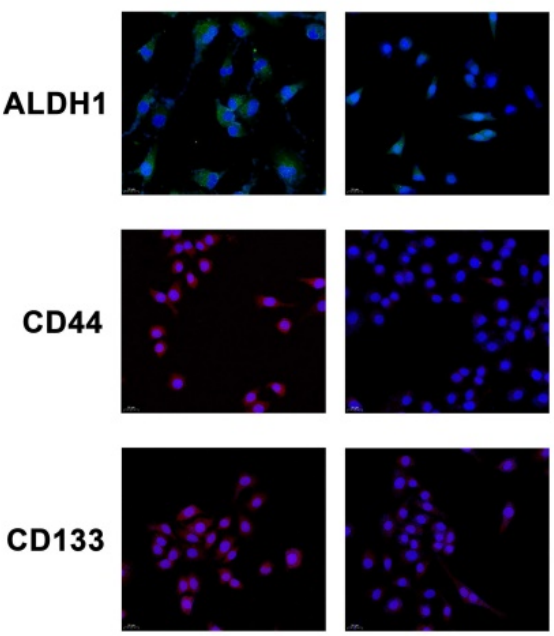
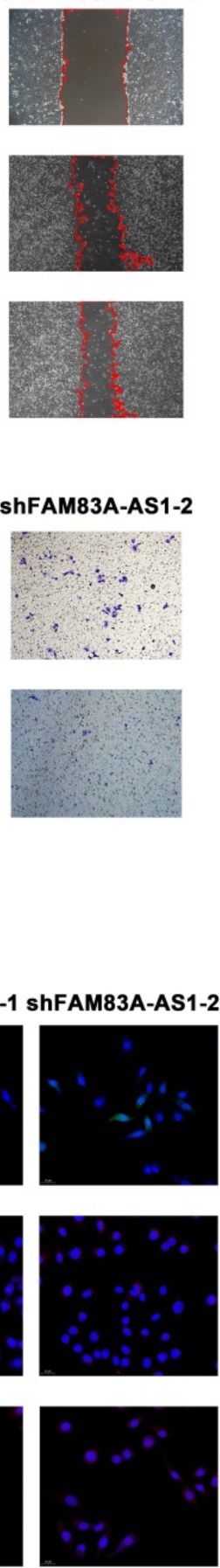

H358

shNC ShFAM83A-AS1-1 ShFAM83A-AS1-2
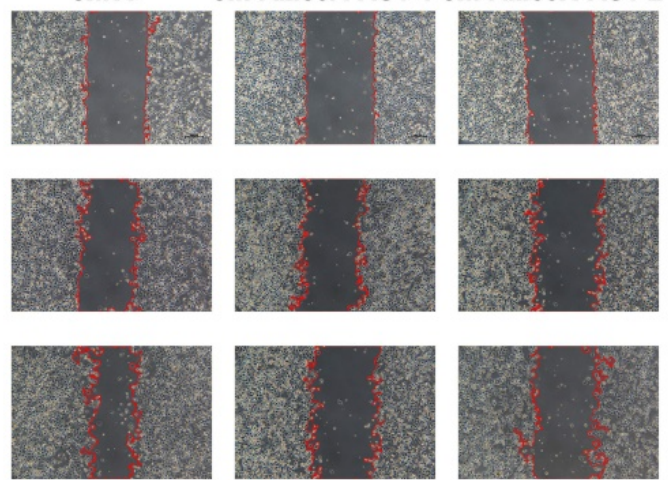

shNC

ShFAM83A-AS1-1 ShFAM83A-AS1-2
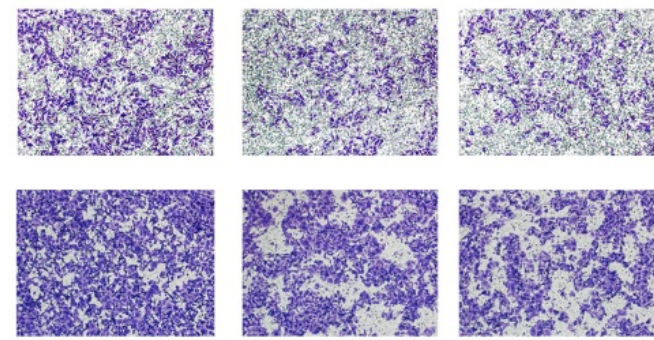

Migration

H358
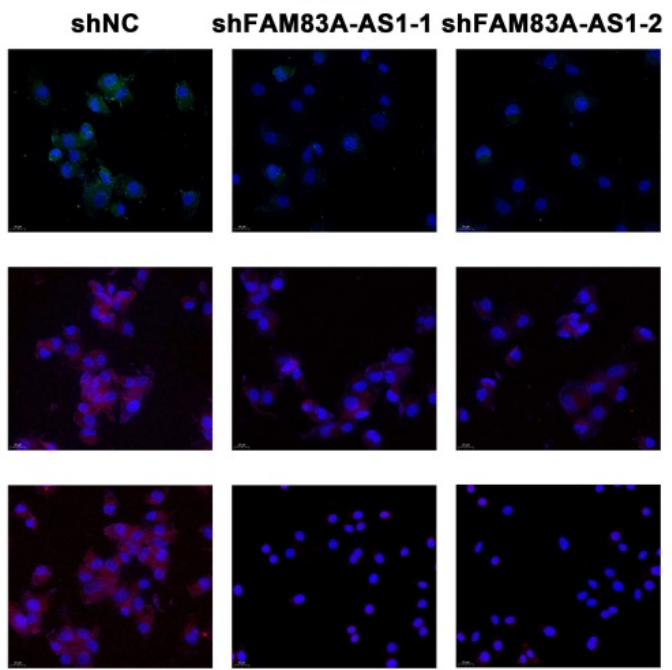

Figure 2. LncRNA FAM83A-AS1 promotes the migration, invasion and stemness of LUAD cell lines under hypoxic conditions in vitro. (a) Wound-healing scratch assays of FAM83A-ASI knockdown and control LUAD cells under hypoxic conditions. (b) Cell migration and invasion assays were performed in FAM83A-AS1 knockdown and control LUAD cells under hypoxic conditions. (c) Fluorescence in situ hybridization of stemness-related genes in FAM83A-ASI knockdown and control LUAD cells under hypoxic conditions.

We further explored the relationship between FAM83A-AS1 and cell stemness. Using quantitative real-time PCR, we measured the expression of cell stemness-related genes in A549 and H358 cell lines 
depleted of or overexpressed with FAM83A-AS1. The results revealed that CD133, CD44, and ALDH1 expression levels were decreased in both cell lines when FAM83A-AS1 was depleted, while they were elevated in the FAM83A-AS1 overexpressed cell lines (Supplementary Figure 2C). In addition, CD33 showed a similar trend in H358 cells but not in A549 cells. Then, to further verify the effect of FAM83A-AS1 on stemness-related genes, we explored the amplification variations of the above genes using fluorescence in situ hybridization (FISH) experiments. Similarly, the results confirmed that amplification of the above genes was enhanced in FAM83A-AS1 overexpressed cells, but little amplification was seen in FAM83A-AS1 knockdown cells (Figure 2C). In addition, flow cytometry also confirmed reduced CD133 expression in FAM83A-AS1 knockdown A549 cells (Supplementary Figure 2D).

Collectively, the above experimental results confirmed that inhibition of lncRNA FAM83A-AS1 expression reduced the invasion and migration abilities of lung adenocarcinoma and FAM83A-AS1 could impact the cell stemness by influencing the expression of CD133, CD44, and ALDH1 in LUAD under hypoxic conditions.

\section{LncRNA FAM83A-AS1 promotes glycolysis in LUAD cell lines in vitro and in vivo and influences the expression of genes related to glucose metabolism}

FAM83A-AS1 has been proven to be involved in cellular metabolic processes such as glycolysis and oxidative phosphorylation previously. To further explore the relationship between FAM83A-AS1 and metabolism, we used the Seahorse assay to determine the metabolic alterations in knockdown or overexpressed FAM83A-AS1 LUAD cell lines. The $\mathrm{O}_{2}$ consumption rate (OCR) showed that the basal respiration of the cells was slightly reduced, while maximal respiratory capacity was significantly inhibited in cells overexpressing FAM83A-AS1 (Figure 3A). The extracellular acidification rate (ECAR) significantly increased glycolysis and glycolytic capacity in cells overexpressing FAM83A-AS1. As expected, the opposite trend was observed in FAM83A-AS1 knockdown cells. The above results suggest that FAM83A-AS1 can inhibit oxidative phosphorylation and promote cellular glycolytic capacity.

Furthermore, to further verify the effects of FAM83A-AS1 on the metabolism in vivo, we subcutaneously injected FAM83A-AS1 overexpressed or knocked down A549 cells into nude mice. After two weeks, PET/CT of the xenografts showed that tumors formed in the FAM83A-AS1 overexpressed group were significantly larger and their metabolic capacities were increased compared to the FAM83A-AS1 knockdown group (Figure 3B). In summary, our study confirms that FAM83A-AS1 can affect tumor metabolism, especially enhancing LUAD cells' glycolysis in vitro and in vivo.

Next, we verified the expression of glucose metabolism-related genes using qRT-PCR in cell lines overexpressing or depleted of FAM83A-AS1. We found that HK2, PKM2, and LDHA were highly expressed in FAM83A-AS1 overexpressing cells, while a down-regulation was observed in FAM83A-AS1 knocked down cells (Figure 3C). Then, we verified the expression of the above three genes and hypoxia-inducible factor 1 subunit alpha () at the protein level. The results demonstrated that FAM83A-AS1 overexpression could promote the protein expression of the above genes, while the opposite results were observed after FAM83A-AS1 knockdown (Figure 3D). Consistently, we observed similar results in patient's samples with high expression of FAM83A-AS1 (Figure 3E). Thus, we hypothesized that FAM83A-AS1 could affect LUAD metabolism by regulating the expression of genes related to glucose metabolism.

\section{LncRNA FAM83A-AS1 binds to HIF- $1 \alpha$ at its $\mathrm{N}$-terminal VHL recognition site, thereby inhibiting HIF-1 a degradation by obstructing the interaction of HIF- I $\alpha$ with VHL}

To further investigate our above results, we used PRIDB (http://bindr.gdcb.iastate.edu/PRIDB)[17] to predict the proteins that can bind to FAM83A-AS1. We found that FAM83A-AS1 was able to bind to HIF-1a with a binding probability of 0.80 . Next, we used catRAPID (http://service.tartaglialab.com/ page/catrapid_group)[18] to predict the potential binding sites between HIF-1a and FAM83A-AS1. The results showed that the binding region was probably located at the 376-527 amino acid sites of HIF-1a (Supplementary Table 1). We found that this locus overlaps with the pVHL binding site. Under normoxia, the oxygen-dependent degradation (ODD) region of HIF-1a is recognized and hydroxylated by PHD enzymes[19, 20]. After hydroxylation, HIF-1a can bind to $\mathrm{pVHL}$ and undergoes ubiquitination and subsequent degradation by the proteasome[21]. Therefore, we hypothesized that FAM83A-AS1 could competitively bind at the binding site of pVHL on HIF-1a, preventing the attachment of pVHL and eventually inhibiting the degradation of HIF-1a. 
A
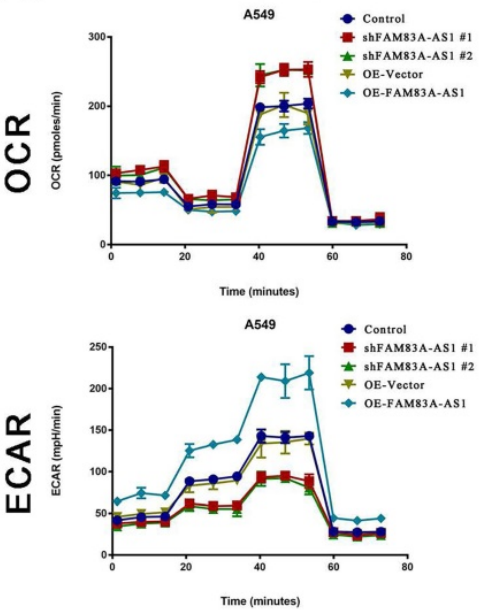

B

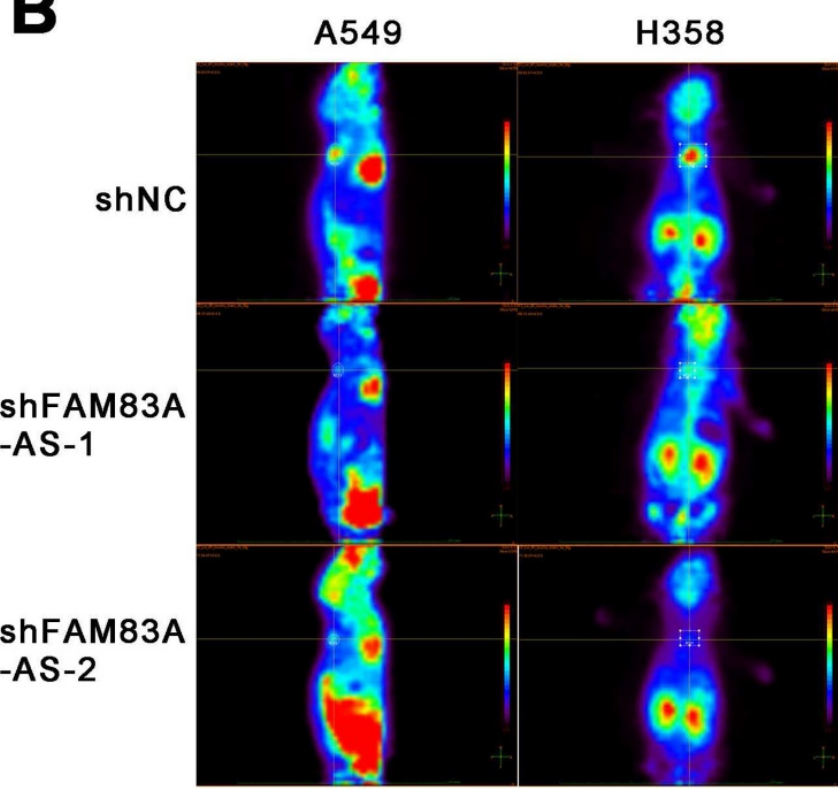

E
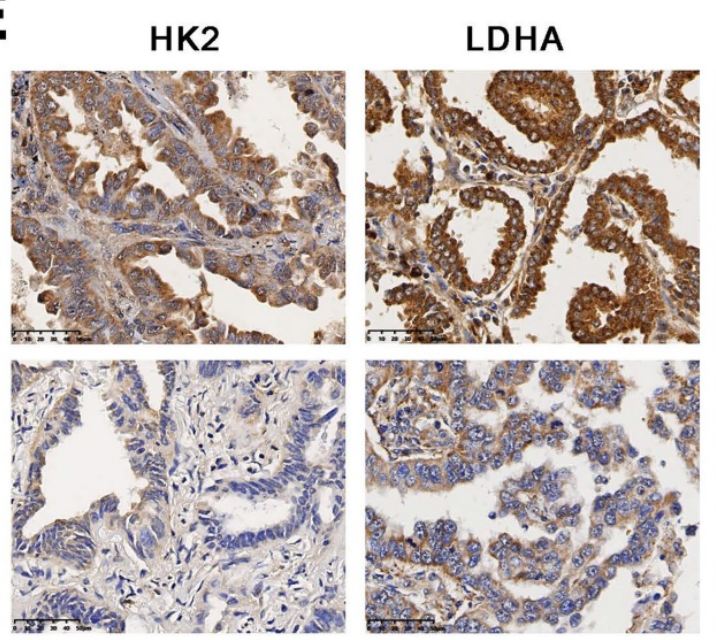

H3538

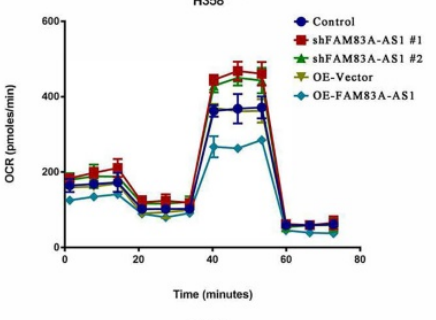

H35

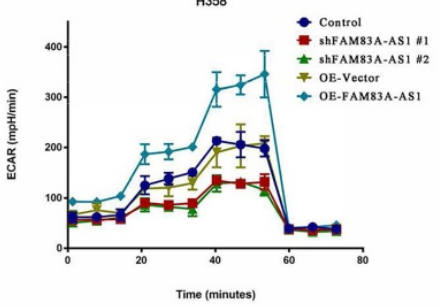

D

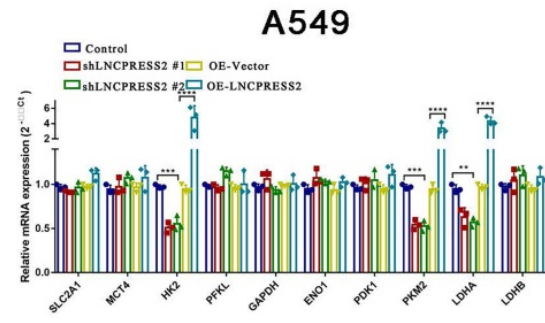

H358
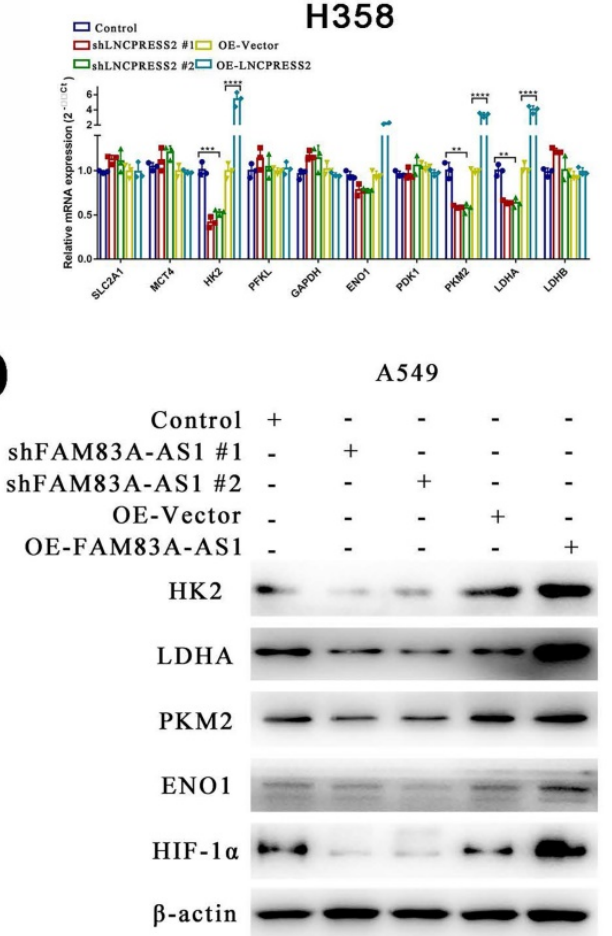

H358

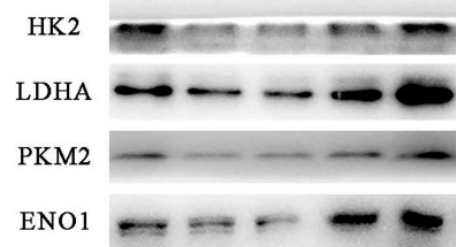

PKM2

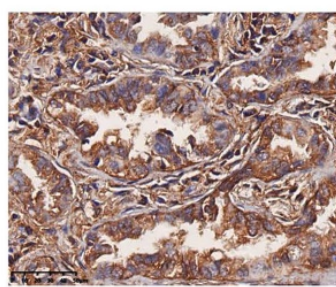

$\mathrm{HIF}-1 \alpha$

$\beta$-actin

\section{FAM83A-AS \\ High Expression}

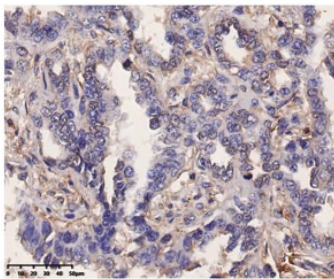

\section{FAM83A-AS \\ Low Expression}

Figure 3. LncRNA FAM83A-ASI promotes glycolysis in LUAD cell lines in vitro and in vivo and influences the expression of genes related to glucose metabolism. (a) The $\mathrm{O} 2$ consumption rate (OCR) and extracellular acidification rate (ECAR) of FAM83A-AS1 knockdown, overexpressing and control cells. (b) PET/CT of mouse model which subcutaneous injected FAM83A-AS1 knockdown and control A549 cells. (c) Relative gene expression(2- $\triangle \triangle C T)$ of glucose metabolism-related genes in FAM83A-AS1 knockdown, overexpressing and control cells. (d) Western blotting detected the expression levels of glucose metabolism-related genes and HIF1A in FAM83A-ASI knockdown, overexpressing and control cells. (e) Immunohistochemistry of glycolysis-related genes expressed in patients of different FAM83A-AS1 expression LUAD. 

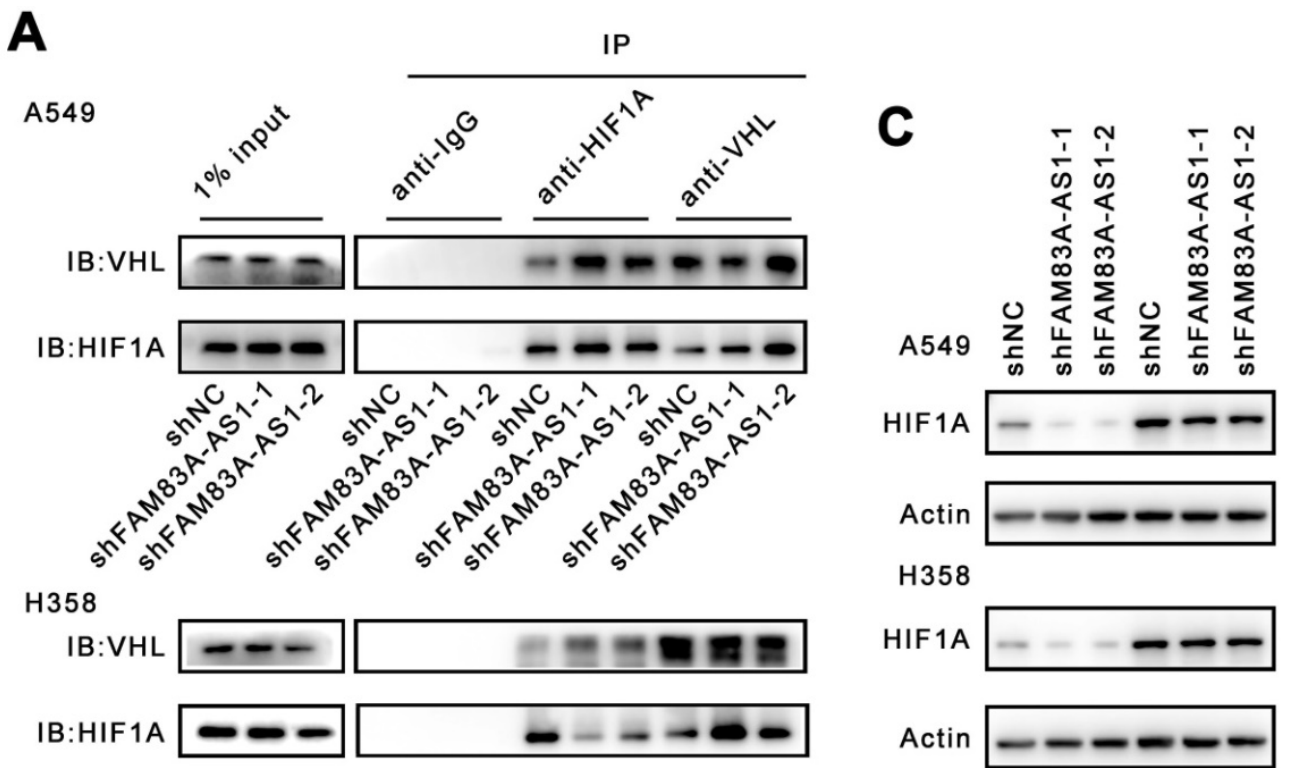

H358
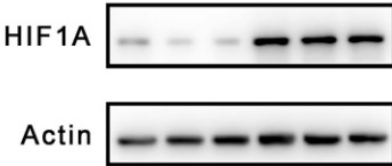

B

A549
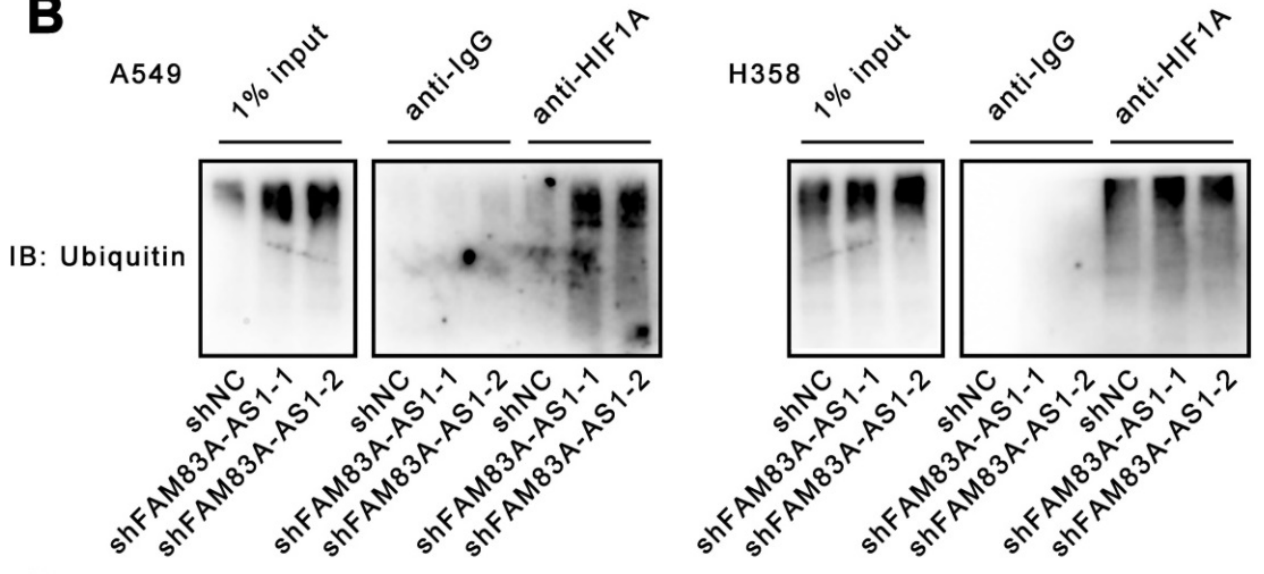

D
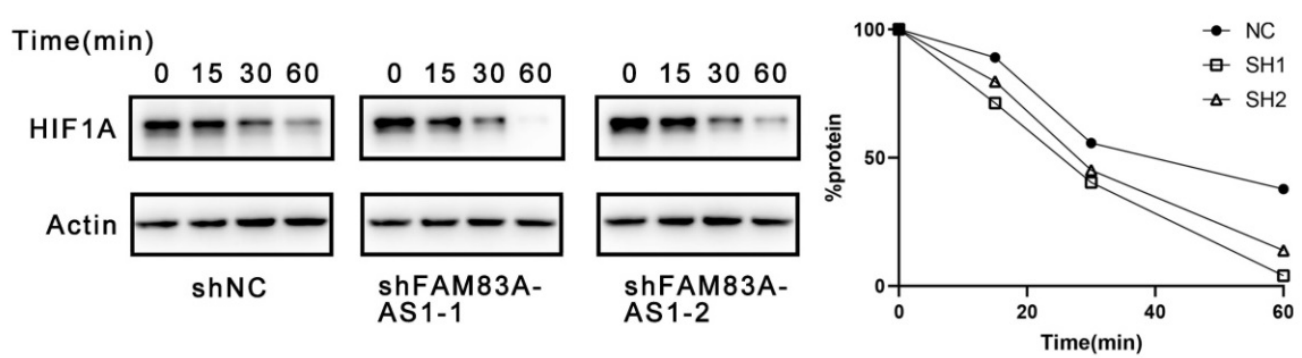

Figure 4. LncRNA FAM83A-ASI inhibits HIF-1 $\alpha$ degradation by obstructing the interaction of HIF-1 $\alpha$ with VHL. (a) Immunoprecipitation of anti-HIF1A and anti-VHL in hypoxia treated A549 and H358 cells knocking down FAM83A-ASI. (b) The ubiquitination level of HIFIA in hypoxia conditions of FAM83A-ASI knockdown and control A549 and H358 cells. (c) The HIF1A expression level was analyzed by western blotting in FAM83A-AS1 knockdown and control LUAD cells after treated with proteasome inhibitor MG132 (10 MM). (d) The HIFIA expression level was analyzed by western blotting in FAM83A-ASI knockdown and control LUAD cells after treated with $25 \mu \mathrm{M} \mathrm{CHX}$ for indicated time periods under normoxic conditions.

Considering that FAM83A-AS1 could attach to the binding site of HIF-1a and VHL, we investigated whether FAM83A-AS1 could affect the interaction of HIF-1a and VHL. Our results demonstrated that FAM83A-AS1 knockdown could promote the interaction of HIF-1a and VHL in LUAD cell lines (Figure 4A). HIF-1a can form a complex with Cullin-RING E3 ubiquitin ligase (CRLs) upon combining with VHL and is subsequently degraded by the proteasome[22-24]. However, under normoxic conditions, HIF-1a is not recognized by VHL and thus accumulates intracellularly. Therefore, we next detected the ubiquitination level of HIF-1a. The results revealed that FAM83A-AS1 knockdown caused a significant increase in the ubiquitination level of HIF-1a (Figure 4B). Subsequently, we treated the A549 and H358 cells with the proteasome inhibitor MG132 and assayed the expression level of HIF-1a. 
We found that MG132 almost eliminated the inhibitory effect of FAM83A-AS1 on HIF-1a (Figure 4C). To investigate whether FAM83A-AS1 affects the stability of HIF-1a, we treated the cells with cycloheximide (CHX), an inhibitor of protein synthesis. Cells were first incubated under hypoxic conditions for 24 hours, followed by the addition of CHX and resumption of normoxic conditions. Compared with normal LUAD cells, the half-life of HIF-1a was significantly shorter in FAM83A-AS1 knockdown cells (t1/2 shNC vs. sh FAM83A-AS1 vs. shFAM83A-AS2 $=37$ vs. 24 v.s $22 \mathrm{~min}, p<0.05$ ) (Figure 4D). In summary, we reveal that FAM83A-AS1 can inhibit the binding of HIF-1a to VHL, weakening VHL-mediated ubiquitination and proteasomal degradation, which in turn leads to the accumulation of HIF-1a.

\section{The effect of LncRNA FAM83A-AS1 on migration and invasion of LUAD cells is regulated by HIF-1 $\alpha$}

Next, we confirmed whether FAM83A-AS1 exerts its biological role through HIF-1a. To that end, we transfected HIF-1a plasmids, whose VHL binding sites have been mutated in FAM83A-AS1 knockdown cells. We found that FAM83A-AS1 knockdown cells transfected with the mutated HIF-1a plasmid had a significantly subdued migration and invasion compared to FAM83A-AS1 knockdown cells alone, with a trend resembling that of normal cells (Figure 5A). This suggests that FAM83A-AS1 promotes the invasion and migration of LUAD cells by mediating the high expression of HIF-1a.

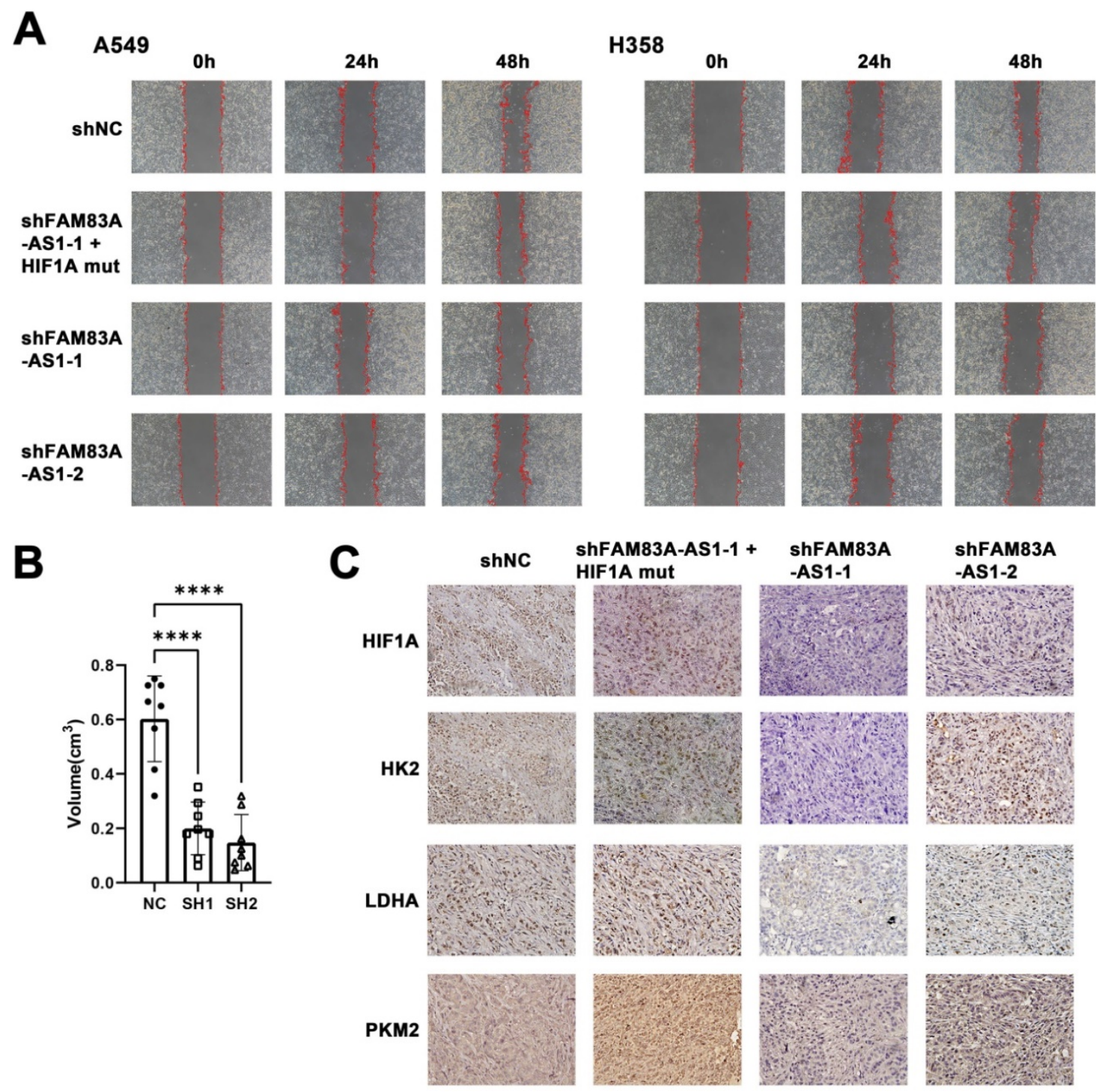

Figure 5. The effect of LncRNA FAM83A-ASI on migration and invasion of LUAD cells is regulated by HIFIA and knockdown LncRNA FAM83A-ASI inhibits tumor growth and suppresses the expression of HIF-1 $\alpha$ and glycolysis-related genes in vivo. (a) Wound-healing scratch assays of FAM83A-AS1 knockdown and control LUAD cells and FAM83A-ASI knockdown cells transfected with the mutated HIF-1a plasmid under hypoxic conditions. (b) Tumor volume size of tumors in subcutaneous mouse model which injected FAM83A-AS1 knockdown and control LUAD cells. (c) Immunohistochemistry of glycolysis-related genes and HIF1A expressed in tumors from subcutaneous mouse model. 
(1) FAM83A-AS1 promotes the expressions of HIF-1a

(2) HIF-1 $\alpha$ increases glycolysis and enhances the proliferation and the migration of cancer cells in Lung adenocarcinoma

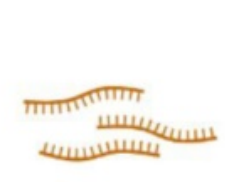

HIF-1 $\alpha$ mRNA

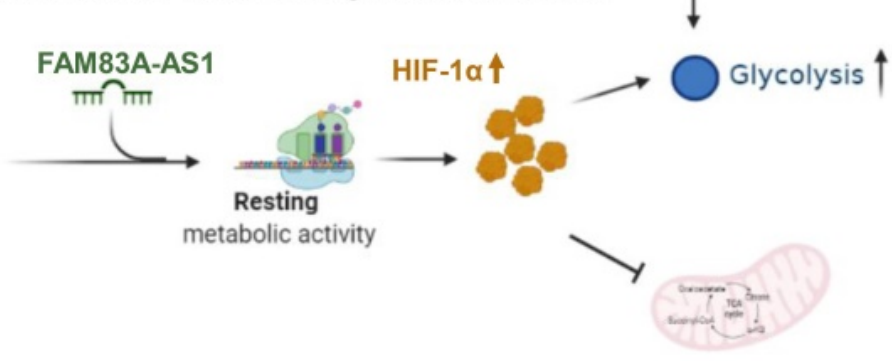

Normal Lung

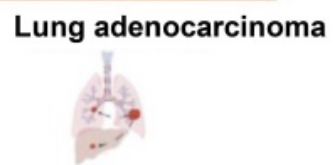

Figure 6. Schematic model of FAM83A-AS1-HIF-1 $\alpha$ signaling axis in LUAD.

\section{Knockdown of LncRNA FAM83A-AS1 inhibits tumor growth and suppresses the expression of HIF- $1 \alpha$ and glycolysis-related genes in vivo}

To investigate if the effects of FAM83A-AS1 would translate in vivo, we used nude mice injected with A549 cells transfected with shFAM83A-AS1 or a control vector. Mice were sacrificed 4 weeks later, and tumors were dissected and measured. The results revealed that the tumor volume of the control group transfected with the blank vector was significantly larger than that of the knockdown FAM83A-AS1 group (Figure 5B, Supplementary Figure 2). Next, the tumor tissues were sectioned and stained, and similarly, the results confirmed high expression of HIF-1a and glycolysis-related genes like LDHA, HK2, PKM2 in the control group and low expression in the shFAM83A-AS1 LUAD (Figure 5C). In conclusion, our results confirmed that FAM83A-AS1 could promote LUAD development in vivo by affecting HIF-1 $\alpha$ and enhancing glycolysis.

\section{Discussion}

Herein, we are first to identify FAM83A-AS1 as a metabolism-related lncRNA, which was found to be highly correlated with glycolysis, hypoxia, and OXPHOS pathways in LUAD patients using bioinformatics analysis. Subsequent functional experiments revealed that FAM83A-AS1 promotes invasion, migration and enhances cell stemness in LUAD cells under hypoxic conditions. In addition, FAM83A-AS1 could promote glycolysis and affect the expression of glycolysis-related genes in vitro and in vivo. Our study further uncovered that FAM83A-AS1 could bind to the N-terminal of HIF-1a to inhibit the interaction between HIF-1a and VHL, thus preventing the degradation of HIF-1a and allowing its stable presence intracellularly. In contrast, transfecting with the mutated HIF-1a plasmid in cells with knockdown of FAM83A-AS1 eliminated the migration and invasion changes. In summary, our study demonstrates that FAM83A-AS1 contributes to LUAD development via the FAM83A-AS1/HIF-1a/ glycolysis axis (Figure 6).

Many studies have reported that lncRNA, FAM83A-AS1 has potent tumor-promoting activity in oesophageal squamous cell carcinoma (ESCC), lung cancer, and liver carcinoma[25-27]. For instance, Xiao $\mathrm{G}$ et al. [28] discovered that FAM83A-AS1 could promote LUAD cell migration and invasion, consistent with our results. In addition, several research showed that IncRNAs play a major role in inhibiting drug resistance and act as mediators involved in different chemoresistance mechanisms[29]. Previous studies confirmed that FAM83A-AS1 could promote the expression of intracellular FAM83A and thus confers tumor cells with EGFR-TKI resistance[30-32], indicating that targeting FAM83A-AS1 might have the potential to reverse EGFR-TKI resistance in LUAD. Overall, these results suggest that FAM83A-AS1 is a potential modulator of cancer driver genes and a prognostic marker across different tumors.

The role of lncRNAs in tumor development and metabolism have received increasing attention[33].For example, the lncRNA MALAT1, UCA1, NBR2, etc. has been reported to regulate tumor cell metabolism, including promotes a glycolytic phenotype and increased lactate production, through regulating metabolic transcription factor, enzymes and relating 
pathways[34-36]. HIF-1a-stabilizing lncRNA (HISLA) from tumor-associated macrophages regulates aerobic glycolysis in breast cancer cells by inhibiting the hydroxylation and degradation of HIF-1a. Thus, the understanding of the role of lncRNAs in metabolism may help provide new therapeutic targets and novel diagnostic and prognosis markers for human cancer.

LncRNAs that take effect via reducing the intracellular transcripts level, or attenuating their activities and molecular functions in malignant cells[37] are potential targets for cancer therapy. For example, MALAT are associated with tumor recurrence after liver transplantation[38], as well as suppresses the progression and metastatic ability of breast cancer cells[39] . H19 is re-expressed during tumorigenesis and promoted tumorigenic properties in broad tissue types including breast, lung, esophageal and bladder[37]. Moreover, a clinical trial from Wuhan Union Hospital on lncRNA as a potential target for lung cancer diagnosis is underway. This clinical trial is mainly based on the identification of early lung-cancer-specific exosomal lncRNA biomarkers to improve the diagnosis rate of early lung cancer[40]. Also, a clinical trial which is related to HOTAIR and thyroid cancer is being processed[41]. Furthermore, numerous studies recent years have demonstrated that lncRNAs play a significant role in resistance of cisplatin and oxaliplatin based chemotherapy [42, 43]. These all imply that targeting lncRNAs may show important clinical implication by selectively affecting disseminated cancer cells or residual cancer cells after surgery. Unfortunately, till now, none of the drugs directly targeting lncRNA were approved for application of anti-tumor therapy.

Moreover, our present study found evidence of the critical role of a specific lncRNA in the positive feedback loop of assembling the HIF-1a transactivation complex, which is similar to previous studies[44]. For instance, lincRNA-p21 was reported as a hypoxia-sensitive lncRNA by influencing the stability of HIF-1a protein. This positive feedback loop reciprocally promoted glycolysis under hypoxia[45]. Another study showed that hypoxia-inducible lncRNA (LncHIFCAR)[46] formed a complex with HIF-1a via direct binding and facilitated the recruitment of HIF-1a to the target promoters, thus was crucial for metabolic shifting and progression of oral carcinoma[47].

On the other hand, although multiple treatment strategies have been used in LUAD patients, the overall prognosis of LUAD remains poor[48, 49], indicating new therapeutic approaches that are more effective in treating LUAD are still warranted. Recently, many studies have demonstrated that understanding metabolic reprogramming of tumor cells is fundamental for understanding tumor drug resistance and developing anticancer therapy[50, 51]. Malignant cells generally obtain energy through glycolysis, which is less efficient rather than mitochondrial oxidative energy metabolism, which produces more energy but produces the byproduct, lactic acid[52,53]. Previous studies demonstrated that the acidic TME could attenuate anti-tumor lymphocytes' ability to suppress tumor activation[54, 55]. The rapid progress of malignant cells combined with abnormal angiogenesis results in hypoxia within the tumor microenvironment, which leads to the accumulation of low oxygen-responsive HIF-1a [56, 57]. Furthermore, upregulation of HIF-1a activates many vital metabolism-related cancer marks such as angiogenesis, glucose metabolism and cell proliferation/viability, which has a crucial role in tumor survival and progression, leading to the increased possibility of metastasis as well as mortality rates clinically[58]. Previous studies proved that selective HIF-1a targeting molecules are highly likely to be the focus of future research[58], including mTOR inhibitors, cardiac glycosides and topoisomerase [59].

Nevertheless, our study has some limitations. Firstly, we did not clarify the specific effects of FAM83A-AS1 on cell proliferation and stemness. Moreover, the regulation mechanism of FAM83A-AS1 on glycolysis-related genes was not particularly defined, which we aim to clarify in our further research. Overall, our study demonstrates that FAM83A-AS1 is a potential biomarker and therapeutic target in LUAD. Importantly, we uncovered that FAM83A-AS1 contributes to LUAD development via the FAM83A-AS1/HIF-1a/ glycolysis axis. Our findings offer new insight into the association between lncRNAs and metabolism remodeling, providing novel targets for cancer therapy.

\section{Materials and methods}

\section{Ethics statement}

Approval for this study was issued by the Ethics Committee of Zhongshan Hospital, Fudan University, China (B2021-137R). Patients gave informed consent upon hospitalization.

\section{Patients and specimens}

Tissue samples were obtained from patients who underwent surgery in the Department of Thoracic Surgery, Zhongshan Hospital, between January 2019 and December 2020 and whose postoperative pathology was confirmed as lung adenocarcinoma. All studies on human specimens were approved by the Ethics Committee of Fudan University Zhongshan Hospital. 


\section{Identification of FAM83A-ASI as a metabolism-related IncRNA with Bioinformatic analysis}

The expression and clinical data of FAM83A-AS1 in TCGA-LUAD patients were downloaded from the TCGA data portal (https://xenabrowser.net/). Maftools package[60] was used to summarized, analyzed, annotated, and visualized the somatic mutations and copy number variants in our research. GSVA package[61] was applied in our study to calculate the metabolic pathway scores. The gene sets of pathways were downloaded from The Molecular Signatures Database (MSigDB) (http://software .broadinstitute.org/gsea/msigdb/index.jsp).

Pathway-level-metabolic gene set enrichment analysis was performed using R Bioconductor package GSVA v1.32.0 function gsva() with parameters "method = gsva, rnaseq $=$ FALSE, abs.ranking $=$ FALSE, min.sz.

\section{Cell culture and lentivirus infection}

The lung adenocarcinoma cell lines (A549, H358) were purchased from the Chinese Academy of Sciences Cell Bank. Cells were cultured in DMEM (Hyclone, UT, USA) supplemented with 10\% fetal bovine serum (Hyclone, UT, USA), 1\% penicillin, and $1 \%$ streptomycin cultured at $37^{\circ} \mathrm{C}$ in a $5 \% \mathrm{CO} 2$ atmosphere and saturated humidity. The hypoxic environment was simulated by $94 \% \mathrm{~N}_{2}, 1 \% \mathrm{O}_{2}$, and $5 \%$ $\mathrm{CO}_{2}$. Two different short hairpin RNAs (shRNA) targeting FAM83A-AS1 were ligated to lentiviral vectors with puromycin resistance, transfected using lipo3000 (Invitrogen, CA, USA), and cell lines were subsequently screened with puromycin.

\section{Quantitative real-time polymerase chain reaction (qRT-PCR)}

RNA was extracted with TRIzol (Invitrogen, CA, USA), and PrimeScript RT Reagent kit (TaKaRa, Tokyo, Japan) was used for reverse transcription. SYBR Green Premix Ex Taq (TaKaRa, Tokyo, Japan) was used to perform qRT-PCR analysis. Relative RNA expression levels were all measured by the QuantStudio 5 Real-Time PCR System (Thermo Fisher Scientific, MA, USA). The primer sequences were provided in supplementary materials.

\section{Western blotting}

Western-blot assay was used to analyze protein expression. The following antibodies were used to assay: anti-HIF-1a (1:1000, \#36169, Cell Signaling Technology), anti-PKM2 (1:1000, \#4053, Cell Signaling Technology), anti-LDHA (1:1000, \#3582, Cell Signaling Technology), anti-Hexokinase II (1:1000, \#ab209847, Abcam), anti-Enolase-1 (1:1000, \#3810, Cell Signaling Technology), anti-VHL (1:1000, \#68547,
Cell Signaling Technology), anti-Ubiquitin (1:1000, \#3936, Cell Signaling Technology), anti- $\beta$-ACTIN (1:3000, \#AF5001, Beyotime). The band intensity was quantified using Image J software (NIH, Bethesda).

\section{Cell migration and invasion assays}

A Transwell system $(8 \mu \mathrm{m}$ pore size, BD Biosciences) was used for cell migration and invasion assays. Cells were seeded in the upper chamber and cultured in a serum-free medium, and the lower chamber was supplemented with serum medium. For cell invasion assay, the bottom of the upper chamber was coated with Matrigel. After 48 hours of cell seeding, cells on the upper surface were swabbed off, followed by fixing, staining, washing of the cells on the lower surface, and photographed for counting. The wound-healing scratch assay was also used to test the ability of cellular migration. Cells were seeded in a six-well plate one day in advance. Cells were scratched using a $20 \mu \mathrm{l}$ pipette tip, and the cells were incubated in a serum-free medium for 72 hours. Scratch wounds were monitored and photographed at 24, 48, and 72 hours. The distance between the two edges of the scratch wound was measured using Image J software.

\section{CCK8 assay}

The required cells were plated in a 96-well plate with 2000 cells per well, the day before the experiment. After the cells adhered, CCK-8 was added to the wells, and the absorbance was measured after incubating for 2 hours at $37^{\circ} \mathrm{C}$. The absorbance was measured after 24,48 , and 72 hours.

\section{Co-IP assays}

Cells were lysed, and proteins were extracted using Cell lysis buffer for Western and IP (\#P0013, Beyotime). We utilized anti-HIF-1a (1:50, \#36169, Cell Signaling Technology) and anti-VHL (1:50, \#68547, Cell Signaling Technology) antibodies for pull-down assays at $4^{\circ} \mathrm{C}$ and 20rpm overnight. Protein $\mathrm{A}+\mathrm{G}$ Agarose (\#P2055, Beyotime) was then added and mixed at $4^{\circ} \mathrm{C}, 20 \mathrm{rpm}$ for 4 hours. The precipitate was centrifuged, washed and mixed with SDS-PAGE sample loading buffer (\#P0015A, Beyotime) and denatured at $100^{\circ} \mathrm{C}$ for $10 \mathrm{~min}$. The rabbit purified IgG was used as a negative control.

\section{Ubiquitination assays}

Cells were treated with the proteasome inhibitor MG132 $(10 \mu \mathrm{M})$ for $8 \mathrm{~h}$. HIF-1a, and its binding protein were pulled down using the Co-IP assay as described above, and ubiquitination levels were detected using anti-Ubiquitin (1:1000, \#3936, Cell Signaling Technology) antibodies. 


\section{Metabolic assays}

The XFe96 metabolic analyzer (Seahorse, Agilent Technologies, Santa Clara, USA) was used to detect the metabolic state of the cells. Distilled water was added to the sensor cartridge the day before the experiment and incubated overnight at $37{ }^{\circ} \mathrm{C}$ in a non-CO2 incubator. A549 and H358 cells were plated on Seahorse XF96 plates (Agilent Technologies) with a density of $5 \times 104$ cells per well overnight. On the day of the experiment, the distilled water was replaced with XF calibration solution in the sensor cartridge and the media in the Seahorse XF96 plates was changed to Assay Media. Sensor cartridge and Seahorse XF96 plates were both incubated for one hour at $37^{\circ} \mathrm{C}$ in a non-CO2 incubator. Glycolytic capacity was measured after injection of Glucose, Oligomycin and 2-DG. Glucose oxygen consumption rate was measured after injection of Oligomycin, FCCP and Rotentone/Antimycin A. Oxygen consumption rates (OCR) and extracellular acidification rates (ECAR) were also measured. Background OCR and ECAR were derived from wells without cells (medium only) and were automatically subtracted by the software.

\section{Immunohistochemistry}

Immunohistochemistry was performed according to standard protocols[62]. Rabbit antiHIF-1a (1:100, \#ab51608, abcam), rabbit antiHexokinase II (1:500, \#ab209847, abcam), rabbit antiLDHA (1:500, \#3582, Cell Signaling Technology), rabbit anti- PKM2 (1:800, \#4053, Cell Signaling Technology) antibodies were used.

\section{Fluorescence in situ hybridization}

The Cy3-labeled CD133, CD44, ALDH1 probe was constructed by RiboBio (Guangzhou, China). Fluorescence signals were generated using a Fluorescence In situ Hybridization Kit (RiboBio, China), and a Nikon A1 confocal laser scanning microscope (Nikon, Japan) was utilized to take pictures. Fluorescence was quantified using ImageJ (ver. 1.32j, NIH).

\section{Flow Cytometry}

Cells and FITC-conjugated mouse anti-human CD133 (5 $\mu \mathrm{L} / 106$ cells; cat. no.: 567033, BD Biosciences) were incubated on ice for 30 minutes. Then, FACSAria III (BD Biosciences) was used to quantify the required cells, and FlowJo software (TreeStar, Woodburn, OR, USA) was used to analyze the results.

\section{Subcutaneous tumor formation assay}

Male BALB/c nude mice (6-weeks-old)
(Shanghai Slac Laboratory Animal Co., Ltd., Shanghai, China) were maintained in laminar flow cabinets. Nude mice were subcutaneously inoculated with $200 \mu \mathrm{L}(1 \times 106)$ A549 cells transfected with a blank vector or shFAM83A-AS1 vector. The mice were sacrificed 4 weeks later under anesthesia, and the tumors were measured and stained in polymethylene for further analysis.

\section{Statistical analysis}

$\mathrm{R}$ software 3.63, SPSS software 25.0, and Graphpad Software 9.0 were used to assess data. Data are presented as the mean \pm standard error of the mean (SEM). Student's t-test was used to compare the differences between two groups. Correlations between two variations were analyzed by Pearson's correlation. Kaplan-Meier survival curves and log-rank test were employed to depict overall survival (OS). The differences of tumor volume among groups were analyzed by analysis of variance (ANOVA), followed by two-tailed Student's $t$ test. The statistical significances of groups are represented as * $p<0.05$, ** $p<0.01,{ }^{* * *} p<0.001$, and $* * * * * 0.0001$.

\section{Supplementary Material}

Supplementary figures and information. https://www.ijbs.com/v18p0522s1.pdf

Supplementary table.

https://www.ijbs.com/v18p0522s2.xlsx

\section{Acknowledgments}

This work was supported by the Shanghai Pujiang Program (2020PJD009) and Training Programme for the Talents of Zhongshan Hospital, Fudan University (Grant No. 2021ZSYQ24).

\section{Author Contributions}

Cheng Zhan, Zongwu Lin, and Fenghao Sun conceived the study. Zhencong Chen, Zhengyang $\mathrm{Hu}$, Qihai Sui, and Yiwei Huang performed most of the analysis and wrote the manuscript. Zhencong Chen and Zhengyang $\mathrm{Hu}$ verified the underlying data. Mengnan Zhao, Qun Wang, and Ming Li collected the tumor samples and analyzed the data. Jiaqi Liang and Tao Lu helped design and manuscript editing. Lijie Tan supervised this study. All authors read and approved the final version of the manuscript.

\section{Data Sharing Statement}

The data used in this study can be obtained by request to S. FH (sun.fenghao@zs-hospital.sh.cn).

\section{Competing Interests}

The authors have declared that no competing interest exists. 


\section{References}

1. WHO report on the global tobacco epidemic 2019. https://apps.who.int/iris/bitstream/handle/10665/326043/9789241516204eng.pdf?ua $=1 ; 2019$.

2. Torre LA, Siegel RL, Jemal A. Lung Cancer Statistics. Adv Exp Med Biol. 2016; 893: 1-19.

3. Lu T, Yang $X$, Huang $Y$, et al. Trends in the incidence, treatment, and survival of patients with lung cancer in the last four decades. Cancer management and research. 2019: 11: 943-53.

4. Li X, Wenes M, Romero P, et al. Navigating metabolic pathways to enhance antitumour immunity and immunotherapy. Nature Reviews Clinical Oncology. 2019; 16: 425-41.

5. Shang R, Wang M, Dai B, et al. Long noncoding RNA SLC2A1-AS1 regulates aerobic glycolysis and progression in hepatocellular carcinoma via inhibiting the STAT3/FOXM1/GLUT1 pathway. Mol Oncol. 2020; 14: 1381-96.

6. Hung $\mathrm{CL}$, Wang $\mathrm{LY}, \mathrm{Yu} \mathrm{YL}$, et al. A long noncoding RNA connects c-Myc to tumor metabolism. Proceedings of the National Academy of Sciences of the United States of America. 2014; 111: 18697-702.

7. Yang B, Zhang L, Cao Y, et al. Overexpression of IncRNA IGFBP4-1 reprograms energy metabolism to promote lung cancer progression. Mol Cancer. 2017; 16: 154

8. Rupaimoole R, Lee J, Haemmerle M, et al. Long Noncoding RNA Ceruloplasmin Promotes Cancer Growth by Altering Glycolysis. Cell reports. 2015; 13: 2395-402

9. Huang X, Pan L, Zuo Z, et al. LINC00842 inactivates transcription co-regulator PGC-1a to promote pancreatic cancer malignancy through metabolic remodelling. Nature communications. 2021; 12: 3830.

10. Liu PJ, Chen YH, Tsai KW, et al. Involvement of MicroRNA-1-FAM83A Axis Dysfunction in the Growth and Motility of Lung Cancer Cells. International journal of molecular sciences. 2020; 21.

11. Xie P, Guo Y. LINC00205 promotes malignancy in lung cancer by recruiting FUS and stabilizing CSDE1. Bioscience reports. 2020; 40 .

12. Liu $\mathrm{Y}$, Liang $\mathrm{G}, \mathrm{Xu} \mathrm{H}$, et al. Tumors exploit FTO-mediated regulation of glycolytic metabolism to evade immune surveillance. Cell Metab. 2021; 33: 1221-33.e11.

13. Kaymak I, Williams KS, Cantor JR, et al. Immunometabolic Interplay in the Tumor Microenvironment. Cancer cell. 2021; 39: 28-37.

14. Kerk SA, Papagiannakopoulos T, Shah YM, et al. Metabolic networks in mutant KRAS-driven tumours: tissue specificities and the microenvironment. Nature reviews Cancer. 2021; 21: 510-25.

15. Bi G, Bian Y, Liang J, et al. Pan-cancer characterization of metabolism-related biomarkers identifies potential therapeutic targets. Journal of translational medicine. 2021; 19: 219.

16. Zhao $\mathrm{M}$, Chen $\mathrm{Z}$, Zheng $\mathrm{Y}$, et al. Identification of cancer stem cell-related biomarkers in lung adenocarcinoma by stemness index and weighted correlation network analysis. Journal of cancer research and clinical oncology. 2020; 146: $1463-72$

17. Lewis BA, Walia RR, Terribilini M, et al. PRIDB: a Protein-RNA interface database. Nucleic Acids Res. 2011; 39: D277-82

18. Armaos A, Colantoni A, Proietti G, et al. catRAPID omics v2.0: going deeper and wider in the prediction of protein-RNA interactions. Nucleic Acids Res. 2021; 49: W72-w9.

19. Gao S, Lu L, Bai Y, et al. Structural and functional analysis of amphioxus HIFa reveals ancient features of the HIFa family. FASEB journal : official publication of the Federation of American Societies for Experimental Biology. 2014; 28 : 1880-90.

20. Huang LE, Gu J, Schau M, et al. Regulation of hypoxia-inducible factor 1alpha is mediated by an O2-dependent degradation domain via the ubiquitin-proteasome pathway. Proceedings of the National Academy of Sciences of the United States of America. 1998; 95: 7987-92.

21. Masson N, Willam C, Maxwell PH, et al. Independent function of two destruction domains in hypoxia-inducible factor-alpha chains activated by prolyl hydroxylation. The EMBO journal. 2001; 20: 5197-206.

22. Frost J, Galdeano C, Soares P, et al. Potent and selective chemical probe of hypoxic signalling downstream of HIF-a hydroxylation via VHL inhibition. Nature communications. 2016; 7: 13312

23. Jaakkola P, Mole DR, Tian YM, et al. Targeting of HIF-alpha to the von Hippel-Lindau ubiquitylation complex by O2-regulated prolyl hydroxylation. Science (New York, NY). 2001; 292: 468-72.

24. Maxwell PH, Wiesener MS, Chang GW, et al. The tumour suppressor protein VHL targets hypoxia-inducible factors for oxygen-dependent proteolysis. Nature. 1999; 399: 271-5

25. Jia J, Li H, Chu J, et al. LncRNA FAM83A-AS1 promotes ESCC progression by regulating miR-214/CDC25B axis. Journal of Cancer. 2021; 12: 1200-11.

26. Wang G, Li X, Yao Y, et al. FAM83A and FAM83A-AS1 both play oncogenic roles in lung adenocarcinoma. Oncology letters. 2021; 21: 297.

27. He J, Yu J. Long noncoding RNA FAM83A-AS1 facilitates hepatocellular carcinoma progression by binding with NOP58 to enhance the mRNA stability of FAM83A. Bioscience reports. 2019; 39 .

28. Xiao G, Wang $\mathrm{P}$, Zheng $\mathrm{X}$, et al. FAM83A-AS1 promotes lung adenocarcinoma cell migration and invasion by targeting miR-150-5p and modifying MMP14. Cell cycle (Georgetown, Tex). 2019; 18: 2972-85.

29. Zhang X, Xie K, Zhou H, et al. Role of non-coding RNAs and RNA modifiers in cancer therapy resistance. Mol Cancer. 2020; 19: 47.
30. Shi $\mathrm{R}$, Jiao $\mathrm{Z}$, $\mathrm{Yu}$ A, et al. Long noncoding antisense RNA FAM83A-AS1 promotes lung cancer cell progression by increasing FAM83A. Journal of cellular biochemistry. 2019; 120: 10505-12.

31. Cipriano R, Bryson BL, Miskimen KL, et al. Hyperactivation of EGFR and downstream effector phospholipase D1 by oncogenic FAM83B. Oncogene. 2014; 33: 3298-306.

32. Richtmann S, Wilkens D, Warth A, et al. FAM83A and FAM83B as Prognostic Biomarkers and Potential New Therapeutic Targets in NSCLC. Cancers. 2019; 11 .

33. Zhang $\mathrm{Y}$, Mao $\mathrm{Q}$, Xia $\mathrm{Q}$, et al. Noncoding RNAs link metabolic reprogramming to immune microenvironment in cancers. J Hematol Oncol. 2021; 14: 169-

34. Malakar P, Stein I, Saragovi A, et al. Long Noncoding RNA MALAT1 Regulates Cancer Glucose Metabolism by Enhancing mTOR-Mediated Translation of TCF7L2. Cancer Res. 2019; 79: 2480-93.

35. Nanni S, Aiello A, Salis C, et al. Metabolic Reprogramming by Malat1 Depletion in Prostate Cancer. Cancers (Basel). 2020; 13: 15.

36. Li Z, Li X, Wu S, et al. Long non-coding RNA UCA1 promotes glycolysis by upregulating hexokinase 2 through the mTOR-STAT3/microRNA143 pathway. Cancer Sci. 2014; 105: 951-5.

37. Li CH, Chen Y. Targeting long non-coding RNAs in cancers: Progress and prospects. The International Journal of Biochemistry \& Cell Biology. 2013; 45: 1895-910.

38. Lai M-c, Yang Z, Zhou L, et al. Long non-coding RNA MALAT-1 overexpression predicts tumor recurrence of hepatocellular carcinoma after liver transplantation. Medical Oncology. 2012; 29: 1810-6.

39. Kim J, Piao H-L, Kim B-J, et al. Long noncoding RNA MALAT1 suppresses breast cancer metastasis. Nature genetics. 2018; 50: 1705-15.

40. Muchnik E, Loh KP, Strawderman M, et al. Immune Checkpoint Inhibitors in Real-World Treatment of Older Adults with Non-Small Cell Lung Cancer. Journal of the American Geriatrics Society. 2019; 67: 905-12.

41. Lim TY, Park S, Kang CH. A Meta-Analysis Comparing Lobectomy versus Segmentectomy in Stage I Non-Small Cell Lung Cancer. The Korean journal of thoracic and cardiovascular surgery. 2019; 52: 195-204.

42. Majidinia M, Yousefi B. Long non-coding RNAs in cancer drug resistance development. DNA Repair (Amst). 2016; 45: 25-33.

43. Huang $\mathrm{H}$, Chen J, Ding $\mathrm{CM}$, et al. LncRNA NR2F1-AS1 regulates hepatocellular carcinoma oxaliplatin resistance by targeting $\mathrm{ABCC} 1$ via miR-363. J Cell Mol Med. 2018; 22: 3238-45.

44. Zheng F, Chen J, Zhang X, et al. The HIF-1a antisense long non-coding RNA drives a positive feedback loop of HIF-1a mediated transactivation and glycolysis. Nat Commun. 2021; 12: 1341.

45. Yang F, Zhang H, Mei Y, et al. Reciprocal regulation of HIF-1a and lincRNA-p21 modulates the Warburg effect. Mol Cell. 2014; 53: 88-100.

46. Shih JW, Chiang WF, Wu ATH, et al. Long noncoding RNA LncHIFCAR/MIR31HG is a HIF-1a co-activator driving oral cancer progression. Nat Commun. 2017; 8: 15874.

47. Hua Q, Mi B, Xu F, et al. Hypoxia-induced IncRNA-AC020978 promotes proliferation and glycolytic metabolism of non-small cell lung cancer by regulating PKM2/HIF-1a axis. Theranostics. 2020; 10: 4762-78.

48. Allemani C, Matsuda T, Di Carlo V, et al. Global surveillance of trends in cancer survival 2000-14 (CONCORD-3): analysis of individual records for 37 513025 patients diagnosed with one of 18 cancers from 322 population-based registries in 71 countries. Lancet (London, England). 2018; 391: 1023-75.

49. Fu F, Zhang Y, Wen Z, et al. Distinct Prognostic Factors in Patients with Stage I Non-Small Cell Lung Cancer with Radiologic Part-Solid or Solid Lesions. Journal of thoracic oncology: official publication of the International Association for the Study of Lung Cancer. 2019; 14: 2133-42.

50. Giovannini C, Salzano AM, Baglioni M, et al. Brivanib in combination with Notch3 silencing shows potent activity in tumour models. Br J Cancer. 2019; 120: 601-11.

51. Liang $Y$, Zhang $D$, Zheng $T$, et al. IncRNA-SOX2OT promotes hepatocellular carcinoma invasion and metastasis through miR-122-5p-mediated activation of PKM2. Oncogenesis. 2020; 9: 54-

52. Nwosu ZC, Pioronska W, Battello N, et al. Severe metabolic alterations in liver cancer lead to ERK pathway activation and drug resistance. EBioMedicine. 2020; $54: 102699$

53. Wettersten HI, Aboud OA, Lara PN, Jr., et al. Metabolic reprogramming in clear cell renal cell carcinoma. Nature reviews Nephrology. 2017; 13: 410-9.

54. Ngwa VM, Edwards DN, Philip M, et al. Microenvironmental Metabolism Regulates Antitumor Immunity. Cancer research. 2019; 79: 4003-8.

55. Certo M, Tsai $\mathrm{CH}$, Pucino V, et al. Lactate modulation of immune responses in inflammatory versus tumour microenvironments. Nature reviews Immunology. 2021; 21: 151-61.

56. Michiels C, Tellier C, Feron O. Cycling hypoxia: A key feature of the tumor microenvironment. Biochim Biophys Acta. 2016; 1866: 76-86.

57. Soni S, Padwad YS. HIF-1 in cancer therapy: two decade long story of a transcription factor. Acta Oncol. 2017; 56: 503-15

58. Soni S, Padwad YS. HIF-1 in cancer therapy: two decade long story of a transcription factor. Acta Oncologica. 2017; 56: 503-15

59. Semenza GL. Hypoxia-inducible factors in physiology and medicine. Cell. 2012: 148: 399-408.

60. Mayakonda A, Lin DC, Assenov Y, et al. Maftools: efficient and comprehensive analysis of somatic variants in cancer. Genome research. 2018; 28: $1747-56$ 
61. Hanzelmann S, Castelo R, Guinney J. GSVA: gene set variation analysis for microarray and RNA-seq data. BMC bioinformatics. 2013; $14: 7$.

62. Niu H, Huang Y, Yan L, et al. Knockdown of SMAD3 inhibits the growth and enhances the radiosensitivity of lung adenocarcinoma via p21 in vitro and in vivo. International journal of biological sciences. 2020; 16: 1010-22. 